level of consciousness were relatively infrequent and grade of hemiparesis were mild with a fair recovery as a rule. (5) Twenty-three of 73 cases $(27.3 \%)$ died of GVD as a direct cause of death among whom mortality of $\mathrm{CH}$ was $70 \%$ which was significantly higher than that of CI which was only $5.9 \%$. (6) In the present series included were those who manifested apoplectic insults in the course of SLE. Among 67 cases of SLE, 5 developed cerebrovascular accidents (7.5\%). All were females under 42 years of age, and the youngest 23. There were no relationship between the duration of SLE and cerebrovascular accidents. Though obscure as the etiologies of CVA 4 of 5 patients had hypertension particularly of diastolic pressure and all manifested an evidence of renal damage such as proteinuria or reduced

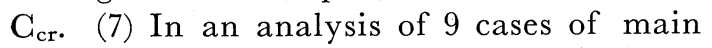
truncal artery occlusion in younger age group etiologies of occlusion were proved to be Moyamoya disease in 4, aortic arch syndrome in 2, atherosclerosis in 1 and unkown in 2. Sites of occlusion were predominantly in internal carotid artery (7 of 9 cases) followed by middle cerebral artery (1) and basilar artery (1). A case of lockedin syndrome and a subclavian steal syndrome associated with middle cerebral artery occlusion were included in this series. The etiologies of these two cases were not exactly known but arteritis could be well suspected.

Concluding remarks: Seventy-three patients under 39 years of age were found among 686 GVD (10.6\%) admitted in the period of 1958 to 1978 . The incidence was in fair agreement with $14.6 \%$ in autopsy materials of 123 cases. In this age group $\mathrm{CH}$ was found to be most frequent with varied underlying diseases such as hypertension, blood disorders, renal diseases and collagen diseases. For this reason the mortality of $\mathrm{CH}$ in this age group proved to be exceedingly higher than that of GVD above 40 years of age. CI v.s $\mathrm{CH}$ ratio as compared in two decade was uninfluenced in GVD of young adults while there was a sharp rise of ratio in those above 40 years of age in accord with general trend of increment in the nation wide surveys. On the contrary mortality of occlusive GVD in young adults was low with a fair functional recovery as a rule. There were varieties of etiologies for the occlusion of cerebral vessels including Moyomoya diseases, aortic arch syndrome, arteritis and so on but a considerable numbers manifested no known etiologies. In main truncal artery occlusion of cerebral vessels atherosclerosis was considered relatively rare and the site of prediction was mostly in internal carotid artery to differ from those above 40 years of age in whom occlusion of middle cerebral artery was most frequent with atherosclerotic origin.

\title{
(4) Diagnostic Problems of Acute Cerebrovascular Stroke
}

\author{
Yoshiaki TAZAKI, MD \\ Department of Internal Medicine, School of Medicine, Kitasato University.
}

The problems related to the diagnosis of acute cerebrovascular stroke have been investigated in the patients who were admitted into the Kitasato University
Hospital.

1. Ghronic subdural hematoma in the old age 
Thirty-three patients with chronic subdural hematome (CSH) were cared for from July, 1971 to March, 1978. This number corresponds to $12 \%$ of 275 patients with cerebral infarction admitted for the same period.

The patients with $\mathrm{CSH}$ were divided into two groups according to age. In eight $(31 \%)$ of 26 patients in the group of over 60 years old, wrong diagnosis of cerebral thrombosis was made by clinical symptoms at the time of admission. However, same erroneous diagnosis was made in only one (14\%) of 7 patients in the group of under 59 years old. This difference may be due to the facts that the patients with $\mathrm{CSH}$ over 60 years old showed atypical clinical symptoms and courses, and that $40 \%$ of them had no apparent history of head trauma.

Eighty percent of the patients with $\mathrm{CSH}$ showed pure motor type of hemiparesis without sensory impairement. Furthermore, facial and hypoglossal nerves were spared in $90 \%$ of them.

\section{Quantitative differential diagnosis of cerebrovascular stroke}

In 1968 Ikeda presented a table of cerebrovascular stroke (CVD-index table) for differential diagnosis among cerebral thrombosis, cerebral hemorrhage and subarachnoid hemorrhage ${ }^{1)}$. According to the external check proved by autopsy, the reliability of the GVD-index table was reported to be $80 \%$. In order to improve the diagnostic accuracy of the table, the Akita Research Institute of Brain and Blood Vessels had presented a new GVDindex table ${ }^{2)}$.

We examined the reliability of the two tables in the study of 43 patients with cerebral hemorrhage and 116 patients with cerebral infarction diagnosed by computed tomography (CT), who were hospitalized from August, 1976 to March, 1978.

As a result of the investigation, exactness of the Ikeda's table was evaluated to be $40 \%$ in cerebral hemorrhage, while the modified table, 58\%. In addition, the correctness for the diagnosis of subcortical hemorrhage by means of either table was as low as $25 \%$. These results indicate that in half of the patients with cerebral hemorrhage diagnosed by CT, correct diagnosis was not obtained by clinical symptoms.

In regard to cerebral hemispheric infarction (102 cases), higher accuracy of $90 \%$ reliability was obtained by the Ikeda's table and $84 \%$ by the modified table.

\section{Hemorrhagic cerebral infarctions}

Twenty-one (24\%) with cerebral supratentorial infarction diagnosed by $\mathrm{CT}$ showed bloody CSF. In six (29\%) of the 21 patients, CT revealed high density areas mixed with those of low density.

The temporal profiles of stroke in the patients with bloody CSF were sudden development of the stroke (76\%), stepwise or gradual development (19\%) and unknown mode of onset (5\%). In another word, $43 \%$ of the patients with sudden development of stroke and $11 \%$ of the patients with stepwise of gradual development showed bloody GSF.

Seventy five percent of the patients who showed bloody CSF with sudden development of stroke was over 60 years old. In $69 \%$ of the patients with bloody CSF, large-sized lesions (larger than $20 \mathrm{~cm}^{2}$ ) were visualized by $\mathrm{CT}$ scanning and in $31 \%$, moderate-sized lesions (larger than $5 \mathrm{~cm}^{2}$ ) were demonstrated. On the other hand, the large-sized, moderate-sized and small-sized lesions (less than $5 \mathrm{~cm}^{2}$ ) were found in $22.5 \%, 25 \%$ and $32.5 \%$ of the patients without bloody CSF, respectively, and no abnormality was observed in the rest of the patients (20\%).

As for prognosis, the group of the patients with bloody CSF showed high mortality rate of $20 \%$. Prognosis for physical function was also poor in these patients and $25 \%$ of them remained bedridden. 
4. The importance of tentorial herniation Severity of acute stroke can be defined by the state of consciousness and clinical findings of brain stem dysfunction. The diagnosis of brain stem dysfunctions due to supratentonial mass lesion have been reported by Plum and Posner ${ }^{3}$.

We have thus studied the relationship between clinical symptoms caused by tentorial herniation and prognosis in 52 patients with supratentorial hemorrhage, who were admitted within 24 hours of stroke. The results of this study were as follows:

All of the 12 patients without signs of tentorial herniation and 11 cases of 24 patients with central syndrome survived by internal treatment and one patient of central syndrome survived by surgically removing hematoma. The others (12 patients) with central syndrome, however, died. Two of 16 patients with uncal syndrome survived, while the others died. Surgery for evacuating hematoma was performed on one of two survivors.

\section{REFERENCES}

1) Ikeda H: Quantitative differential diagnosis of cerebrovascular diseases, Fukuoka Medical Journal, 59 : 818, 1968.

2) Kutsuzawa $\mathrm{T}$ and Tagawa $\mathrm{K}$ : Emergent medical care of stroke, p 35, Kanehara, Tokyo, 1978.

3) Plum $F$ and Posner JB: Diagnosis of stupor and coma, Ed 2, FA Davis, Philadelphia, 1972.

\title{
(5) Correlation of Computed Tomography and Autopsy Findings of Cerebrovascular Diseases
}

\author{
Hitoshi Fukasawa, MD \\ Department of Pathology, Research Institute for Brain and \\ Blood Vessels, Akita
}

Computed tomography (CT) is now widely recognized as extremely informative in the diagnosis of intracranial diseases. To evaluate the accuracy of this new method of examination, however, neuropathologic correlative study is of particular importance. This paper discusses briefly the correlation of CT with neuropathologic findings of cerebrovascular diseases on the basis of more than 80 stroke cases, (40 cases of cerebral hemorrhage, 25 subarachnoid hemorrhage, and 15 cerebral infarction) examined antemortem using the EMI 1010 scanner with $160 \times 160$ matrix and autopsied by the author at our institute.

\section{TECHNICAL CONSIDERATIONS}

Apart from the technological aspects of CT, only two items concerning the braincutting procedure will be mentioned here.

The brains are fixed for 3 weeks in $20 \%$ formalin to avoid distortion and sectioned in $6-12 \mathrm{~mm}$ thick slices as close to the planes of the CT as possible. Then the gross and microscopic findings of these sections are compared with their corresponding $\mathrm{CT}$ slices two-dimensionally to evaluate the accuracy of CT images. It should be noted, however, that this direct comparison is, in fact, only for convenience; and that three-dimensional reconstruction of both the CT and the neuropathologic findings of the brain is essential to a perfect analysis. Taking these into account, the 